\title{
The Human Microbiome Project
}

\author{
Lal Rup
}

Published online: 11 September 2011

(C) Association of Microbiologists of India 2012

The Human Microbiome project (National Institute of Health, USA) has just been completed and information generated from this project was published on June 14, 2012 in Nature and PLoS journals. This project, which consists of a consortium of scientists from nearly 80 universities and scientific institutions, aims to reveal the myriad of microbial organisms residing in healthy humans and related research across various body habitats. The results published in Nature and PLoS journals are considered to be a major milestone in the history of biological success. The presence of more than 10,000 microbial species in human ecosystem reveals the massive contribution of these microbes in controlling various metabolic functions. While the human genome contains nearly 22,000 protein-coding genes, the human microbiome contributes nearly 8 million protein of these bacteria that inhabit the human body. The data has revealed some interesting information. Our body contains 100 trillion bacteria, and the gut is generally not packed with food but with microbes. It is also becoming apparent that the human microbiome contains more number of beneficial than harmful microbes, as the former keeps a check on pathogenic microbes. The potential benefits of the resident commensal microbes also include the control of obesity, brain development deriving nutrition, the ability of the human body to develop resistance towards pathogens and invoking immune response against infections. However, the diversity and distribution of the human microbiota varies systematically across body habitats. While some locations, like the gut, mouth and skin harbour more diverse communities, others show a deficient biogeographical localization of such communities in the

L. Rup $(\bowtie)$

Department of Zoology, University of Delhi, Delhi, India

e-mail: ruplal@gmail.com human body. Likewise, it has been estimated that around 500-1000 species of bacteria inhabit the skin, where they have been studied to play a significant role in educating our immune system to respond to infections and injury, as well as to maintain homeostatic control of skin inflammation. Interestingly, researchers found that the bacterial species of the vaginal microbiome undergo a major shift in preparation for birth, and the passage of the child through the birth canal provides it with its first dose of microbes Major momentum to research in this field was provided when a deliberate alteration of these commensal bacterial populations (availability of germ-free systems) by way of antibiotic treatments was observed to not only increase the rate of allergic reactions, but also lead to many diseases. Further characterization of the factors that influence the diversity and distribution of these commensal bacteria may tie together the research done in the field of medicine to prevent infections, as well as provide a helping hand in therapeutics. As is rightly said, microbiota is indeed acting as 'brothers in arms' in the fight against infectious diseases by endowing us with traits essential for optimal immune responses.

Even though the regulatory mechanism by way of which these bacteria face the pathogens is not well known till now, further ventures into this field will definitely illuminate newer ways to promote better human health and immunity to several infections. This microbiome data cannot be easily extrapolated to more diverse microbiomes of Indian population. Thus there is a need to initiate a parallel study of analyzing human microbiome from individuals from the Indian continent so as to deeply understand the relationship between an individual's physiology and environmental microbiology. 